\title{
Adaptive Energy Selective Active Contour with Shape Priors for Nuclear Segmentation and Gleason Grading of Prostate Cancer
}

\author{
Sahirzeeshan Ali ${ }^{1}$, Robert Veltri ${ }^{2}$, Jonathan I. Epstein ${ }^{2}$, \\ Christhunesa Christudass ${ }^{2}$, and Anant Madabhushi ${ }^{1, \star}$ \\ 1 Rutgers, The State University of New Jersey, New Brunswick, NJ, USA \\ 2 The Johns Hopkins Hospital, Baltimore, Maryland, USA
}

\begin{abstract}
Shape based active contours have emerged as a natural solution to overlap resolution. However, most of these shape-based methods are computationally expensive. There are instances in an image where no overlapping objects are present and applying these schemes results in significant computational overhead without any accompanying, additional benefit. In this paper we present a novel adaptive active contour scheme (AdACM) that combines boundary and region based energy terms with a shape prior in a multi level set formulation. To reduce the computational overhead, the shape prior term in the variational formulation is only invoked for those instances in the image where overlaps between objects are identified; these overlaps being identified via a contour concavity detection scheme. By not having to invoke all 3 terms (shape, boundary, region) for segmenting every object in the scene, the computational expense of the integrated active contour model is dramatically reduced, a particularly relevant consideration when multiple objects have to be segmented on very large histopathological images. The AdACM was employed for the task of segmenting nuclei on 80 prostate cancer tissue microarray images. Morphological features extracted from these segmentations were found to able to discriminate different Gleason grade patterns with a classification accuracy of $84 \%$ via a Support Vector Machine classifier. On average the AdACM model provided $100 \%$ savings in computational times compared to a non-optimized hybrid AC model involving a shape prior.
\end{abstract}

\section{Introduction}

Active Contours (AC) can be categorized as boundary-based (first generation) and region-based (second generation) schemes [1-3]. Most AC models are not intrinsically capable of handling object occlusion or scene clutter. Therefore, the integration of shape priors into the variational formulation represents a natural way to overcome occlusion. Third generation (hybrid) AC models involve

\footnotetext{
* Thanks to funding agencies: National Cancer Institute (R01CA136535-01, R01CA140772 01, R03CA143991-01), and The Cancer Institute of New Jersey.
} 
combining a shape prior with geometric/geodesic active contours that simultaneously achieves registration and segmentation [4 6]. Rousson et al. [5] proposed a novel approach for introducing shape priors into level set representations, focused on $2 \mathrm{D}$ closed shapes. A limitation of most third generation AC models, however, is that only one pair of overlapping objects can be accurately resolved at a time. Further, most of these methods are sensitive to model initialization and typically require varying degrees of user intervention [1-6]. Moreover, the efficiency of these hybrid schemes are limited by the computational overhead of the non linearity of the convergence of the evolving curve. Additionally, the shape prior (the most computationally heavy term in the variational formulation) is typically invoked in segmenting every object in the scene, regardless of whether or not an overlap exists. Non-overlapping objects, in most cases, can be segmented by first and second generation AC models alone.

In this paper, a variational adaptive segmentation scheme (AdACM) is presented. AdACM is fully automated and provides concurrent segmentation of all the overlapping and non overlapping objects in the image. Most of the shape based models reported in literature are only able to handle the overlap resolution of a single pair of objects per image. To decrease the user intervention, we propose to automatically initialize our segmentation scheme via the popular Watershed method. To reduce the computational expense of the model, we selectively invoke the shape prior term, only when overlapping objects need to be resolved and segmented. To do this we leverage a technique based on concavity detection [8], to detect the number of overlapping objects, and hence selectively invoke the appropriate energy functionals (see Figure 1). Note that the complexity of the concavity detection scheme [8] is significantly smaller compared to invoking the shape prior.

The Gleason score (obtained by summing the primary and secondary grades of prostate cancer ( $\mathrm{CaP})$ in the tissue specimen) is the single most important

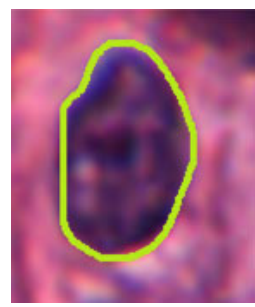

(a)

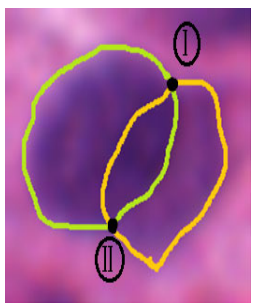

(b)

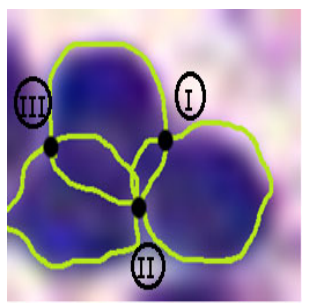

(c)

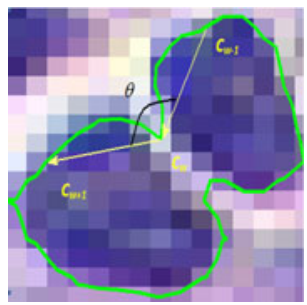

(d)

Fig. 1. Selective incorporation of variational terms based on detection of concavity points. In (a) the absence of concavity point reflects the presence of a single nucleus. Similarly (b) and (c) detection of the number of concavity points represents the number of nuclei. (d) Concavity detection: Three consecutive points on $s\left(c_{w-1}, c_{w}\right.$ and $\left.c_{w+1}\right)$ are used to define two vectors (shown with arrows). The angle $\theta$ between them is a measure of concavity/convexity of the point $c_{w}[8]$. 
prognostic factor in Cap, determined using the glandular and nuclear architecture and morphology within the tumor [7]. In recent years, computerized image analysis methods have been studied in an effort to overcome the subjectivity of conventional grading system. An important prerequisite to such a computerized CaP grading scheme, however, is the ability to accurately and efficiently segment histological structures (glands and nuclei) of interest. In this work, we leverage the AdACM scheme for automatic segmentation of all nuclei on large digitized tissue microarrays (TMAs) of CaP. Additionally, we leverage previous research that has demonstrated a link between nuclear morphology and gleason grade [7] to develop a nuclear morphology based classifier to predict Gleason grade. The accuracy of this classifier is also implicitly reflective of the performance of AdACM, since accurate nuclear segmentation is a pre-requisite for accurately quantifying nuclear morphology.

\section{Hybrid Active Contour Model}

The contours that segment the nuclear-boundaries are represented using the level set method, and are evolved by minimizing the variational energy functional. Under the level set framework, the contour is represented implicitly as the zero level of a higher dimensional embedding function, and the contour propagation is performed by evolving the embedding function. This enables handling topological changes of the boundary (splitting and merging) easily.

\subsection{Shape Term - F shape}

We combine the shape prior, $\psi$, with a Geodesic Active Contour (GAC) to create the shape functional. $\psi$, is created using the statistical methods described in [5]. Each shape in the training sample is embedded as the zero level set of a higher dimensional surface. The Signed Distance Function (SDF) is used to encode the distance between the level set (shape contour) and the grid pixels. The level set formulation of the shape functional is expressed as:

$$
F_{\text {shape }}=\int_{\Omega}(\phi(\mathbf{x})-\psi(\mathbf{x}))^{2}|\nabla \phi| \delta(\phi) d \mathbf{x}
$$

where $\{\phi\}$ is a level set function, $\psi$ is the shape prior, $\delta($.$) is the Dirac function,$ and $\delta(\phi)$ is the contour measure on $\{\phi=0\}$. Equation 1 introduces a shape prior in such a way that only objects of interest similar to the shape prior can be recovered, and all unfamiliar image structures are suppressed. It evaluates the shape difference between the level set $\phi$ and the the shape prior $\psi$ at each iteration of the evolution. However, this formulation only solves for a single level set consistent with the shape prior.

\subsection{Region Homogeneity Term}

We define a functional to drive the shape functional towards a homogeneous intensity region corresponding to the shape of interest. The functional $F_{\text {region }}$ 


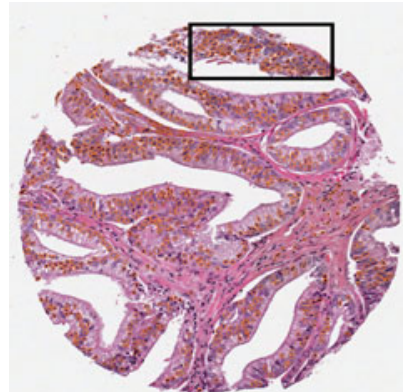

(a)

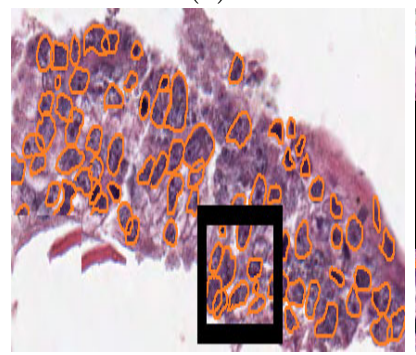

(d)

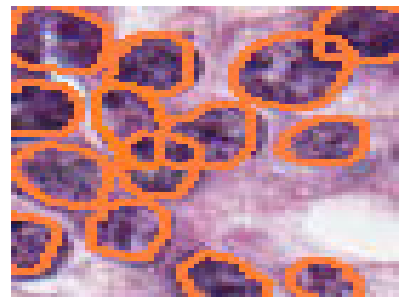

(g)

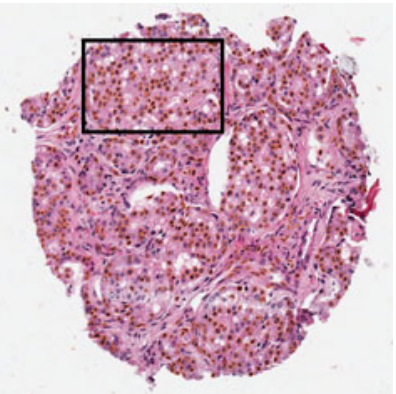

(b)

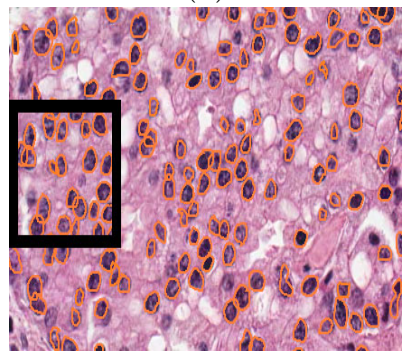

(e)

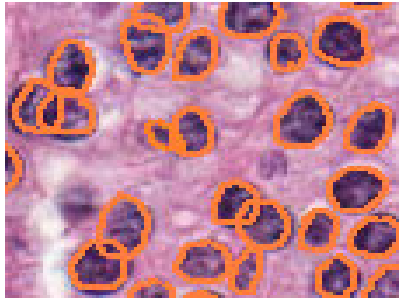

(h)

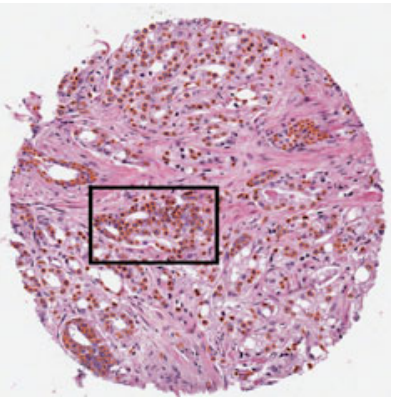

(c)

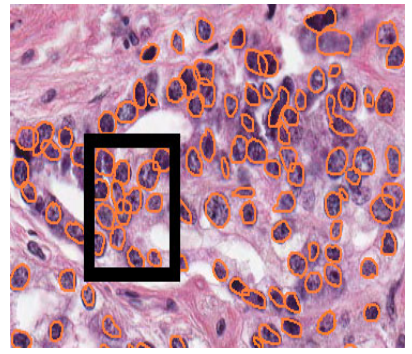

(f)

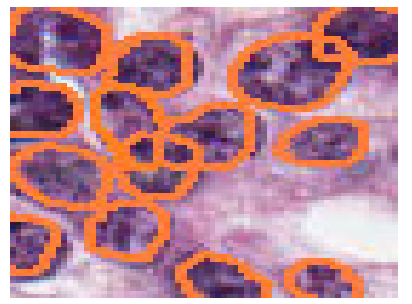

(i)

Fig. 2. (a)-(c) Nuclear segmentations (orange boundaries) for three different TMA cylinders corresponding to Gleason scores 6, 7 and 9. (b)-(f) Magnified ROI's from (a)(c), respectively, reveal that our hybrid ACM (AdACM) with a selective shape prior is able to accurately segment almost all nuclei. (g)-(i) Further magnification of the ROI shown in (a), (b), (c) reveals that our model is able to accurately resolve overlaps and intersections.

can be written with the shape function $\psi$ and statistics of partitioned foreground and background regions, $u_{\text {in }}, u_{\text {out }}$ :

$$
F_{\text {region }}\left(\psi, u_{\text {in }}, u_{\text {out }}\right)=\int_{\Omega} \Theta_{\text {in }} H_{\psi} d \mathbf{x}+\int_{\Omega} \Theta_{\text {out }} H_{-\psi} d \mathbf{x},
$$

where $\psi$ is the shape function, $H($.$) is the Heaviside function, \Theta_{r}=\left|I-u_{r}\right|^{2}+$ $\mu\left|\nabla u_{r}\right|^{2}$ and $r \in\{$ in, out $\}$. 


\subsection{Combining Shape, Boundary and Region-Based Functionals}

We combine $F_{\text {shape }}$ and $F_{\text {region }}$ into a variational formulation:

$$
F=\underbrace{\beta_{s} \int_{\Omega}(\phi(\mathbf{x})-\psi(\mathbf{x}))^{2}|\nabla \phi| \delta(\phi) d \mathbf{x}}_{\text {Shape+boundaryforce }}+\underbrace{\beta_{r} \int_{\Omega} \Theta_{i n} H_{\psi} d \mathbf{x}+\int_{\Omega} \Theta_{\text {out }} H_{-\psi} d \mathbf{x}}_{\text {Regionforce }}
$$

where $\beta_{s}, \beta_{r}>0$ are constants that balance contributions of the boundary based shape prior and the region term. This is an extension of the work of Chen et al in [6]. This formulation effectively integrates shape prior with local and regional intensity information into an unified variational formulation.

\subsection{Segmenting Multiple Objects under Mutual Occlusion}

The level set formulation in Equation (3) is limited in that it allows for segmentation of only a single object at a time. In this work, we incorporate the method presented in [9] into Equation 3. Consider a given image consisting of multiple objects $\left\{O_{1}, O_{2}, \cdots, O_{n}\right\}$ of the same shape. For the problems considered in this work (nuclei segmentation on histopathology images), all nuclei are assumed to be roughly elliptical in shape. Instead of partitioning the image domain into mutually exclusive regions, we allow each pixel to be associated with multiple objects or the background. Specifically, we try to find a set of characteristic functions $\chi_{i}$ such that: $\chi_{i}(\mathbf{x})=\left\{\begin{array}{ll}1 & \text { if } \mathbf{x} \in O_{i} \\ 0 & \text { otherwise. }\end{array}\right.$ We associate one level set per object in such a way that any $O_{a}, O_{b}, a, b \in\{1,2, \cdots, n\}$ are allowed to overlap with each other within the image. These level set components may both be positive on the area of overlap, and enforce the prior on the shapes of objects extracted from the image.

We consider a case of segmenting two objects within an input image. Given an image with two similarly shaped objects $O_{a}, O_{b}, a, b \in\{1, \cdots, n\}$, and for simplicity, assume that they are consistent with the shape prior $\psi$. Then simultaneous segmentation of $O_{a}, O_{b}$ with respect to $\psi$ is solved by minimizing the following modified version of Equation (3):

$$
\begin{aligned}
F\left(\Phi, \Psi, u_{\text {in }}, u_{\text {out }}\right) & =\sum_{i=1}^{N=2} \int_{\Omega}\left(\phi_{i}(\mathbf{x})-\psi(\mathbf{x})\right)^{2}\left|\nabla \phi_{i}\right| \delta\left(\phi_{i}\right) d \mathbf{x}+\beta_{r} \int_{\Omega} \Theta_{\text {in }} H_{\chi_{1} \vee \chi_{2}} d \mathbf{x} \\
& +\int_{\Omega} \Theta_{\text {out }}-H_{\chi_{1} \vee \chi_{2}} d \mathbf{x}+\omega \int_{\Omega} H_{\chi_{1} \wedge \chi_{2}} d \mathbf{x}+\sum_{i=1}^{N=2} \int_{\Omega}\left(\phi_{i}-\psi_{i}\right)^{2} d \mathbf{x}
\end{aligned}
$$

with $H_{\chi_{1} \vee \chi_{2}}=H_{\psi_{1}}+H_{\psi_{2}}-H_{\psi_{1}} H_{\psi_{2}}, H_{\chi_{1} \wedge \chi_{2}}=H_{\psi_{1}} H_{\psi_{2}}$ where $\Phi=\left(\phi_{1}, \phi_{2}\right)$ and $\Psi=\left(\psi_{1}, \psi_{2}\right)$. The fourth term penalizes the overlapping area between the two segmenting regions, and prevents the two evolving level set functions from becoming identical. Minimizing Equation 4 iteratively with respect to dynamic variables, yields the associated Euler-Lagrange equations. The above model can be adapted for $N$ objects (proof not shown). 


\section{Selectively Invoking Energy Terms in Hybrid ACM}

\subsection{Watershed Based Initialization and Concavity Detection}

We use the popular watershed transformation to obtain the initial delineations of nuclear boundaries in the entire image. By creating the binary mask of the delineations, we obtain the estimated boundaries of the nuclei present.

High concavity points are characteristic of contours that enclose multiple objects and represent junctions where object intersection occurs (Figure 1(d)). The area $\mathcal{A}(s)$ of the closed sub-contour $s$ is compared to predetermined area of an ideal nucleus $\tau_{A}$ (empirically set to $\tau_{A}=33$ ). Hence a sub-contour is eligible for a split if $\mathcal{A}(s)>\tau_{A}$. Since $c=(x, y)$, the difference between any two points $c_{w}$ and $c_{w-1}$ will represent a vector in $2 \mathrm{D}$. Concavity points are detected by computing the angle between vectors defined by three consecutive points $\left(c_{w-1}, c_{w}, c_{w+1}\right) \in s$. The degree of concavity/convexity is proportional to the angle $\theta\left(c_{w}\right)$ as shown in Figure $1(\mathrm{~d}) . \theta\left(c_{w}\right)$ can be computed from the dot product relation (Equation 5):

$$
\theta\left(c_{w}\right)=\pi-\arccos \left(\frac{\left(c_{w}-c_{w-1}\right) \cdot\left(c_{w+1}-c_{w}\right)}{\left\|\left(c_{w}-c_{w-1}\right)\right\|\left\|\left(c_{w+1}-c_{w}\right)\right\|}\right) .
$$

\subsection{Adaptive Selection of Energy Functionals in Hybrid AC Model}

Number of detected concavity points, $c_{w} \leq 1$, indicates presence of a single non overlapping nucleus. In such cases, shape prior constraint is not necessary and we reduce the model to only employ the region term by setting $\beta_{s}=0$. Similarly, $l$ number of $c_{w}$ indicate the presence of $l$ overlapping objects. Hence in those regions we initialize the model with the integrated hybrid model (region, boundary, shape terms) with $l$ level sets and set $N=l$ (in Equation 4). Figure 3 illustrates the work flow from initialization to final segmentation for AdACM.

\section{Experimental Design and Results}

Since it is not feasible to evaluate AdACM quantitatively on a per nucleus basis (manual annotation not practical for thousands of nuclei), AdACM was instead evaluated in terms of the ability of the morphologic descriptors extracted from the nuclear boundaries (based off [7]) to distinguish different Gleason patterns of CaP from a total of 40 (2 images per study) TMA images obtained from prostate needle core biopsy samples. The 40 studies comprised 13 Gleason patterns 6 $(3+3), 8$ pattern $7(3+4), 7$ pattern $8(4+4)$ and 5 pattern $9(4+5)$ studies where the first number in the parenthesis refers to the primary and the second number to the secondary Gleason grade. Additionally the ability of the model to selectively invoke energy terms in the variational functional was also evaluated in terms of the (a) overlaps between nuclei resolved (for a randomly selected subset of nuclei) and (b) computational savings over a hybrid model without selective invocation of the shape term. 


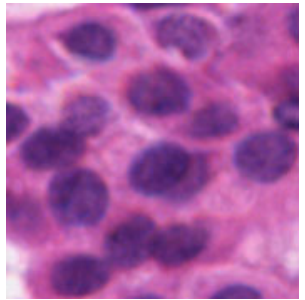

(a)

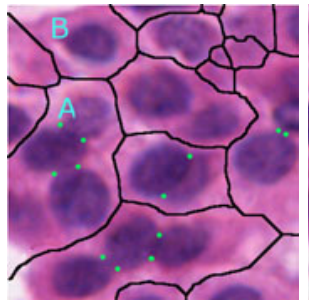

(b)

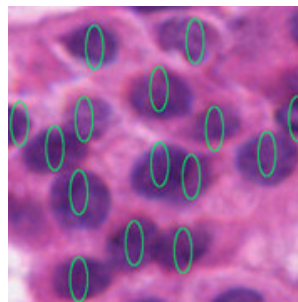

(c)

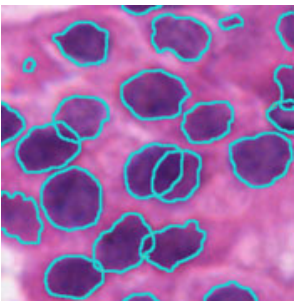

(d)

Fig. 3. Constituent modules of the AdACM. (a) Original image; (b) Watershed segmentation of individual nuclei with an overlay detected concavity points; (c) Placement of initial level sets in the image; (d) final segmentation. Note that in the region shown as A where 4 concavity points where detected, 3 level sets were initialized with the shape prior, whereas in region $\mathrm{B}$, only a single level set (only region based term) was initialized.

\subsection{Discriminating Gleason Patterns Based off Nuclear Morphology}

A total of 7 nuclear features from each of the segmented nuclei were extracted (Morphologic features include: Area Overlap Ratio, Average Radial Ratio, Compactness, Convexity, Mean Nuclear Area, Mean Nuclear Perimeter, Mean Nuclear Diameter). We apply PCA to this feature set to visualize the arrangement of different Gleason patterns in the reduced embedding space. Figure 4(a) illustrates the PCA representation of the 7 morphologic features averaged over each of 40 studies (total of 80 images) and reveals a clear separation between Gleason patterns 3 and 4. Similarly, by exposing the labels for the Gleason scores for each of 40 studies (Figure 4(b)), one can appreciate the separation between Gleason patterns $6,7,8$, and 9 . Note that the PCA plots suggest that the nuclear shape features are able to capture the subtle morphologic differences between the different Gleason patterns, in turn reflecting the accuracy of AdACM. The separation of the intermediate primary grade 3 and grade 4 tumors in the reduced PCA space was also quantitatively evaluated using a support vector machine (SVM) classifier. For a database of 80 images, the SVM classifier achieved an accuracy of $83.8 \pm 0.4 \%$ in distinguishing primary grade 3 from 4 . Classifier evaluation was done via a 3 fold cross validation scheme, over 10 successive runs.

\subsection{Evaluating Overlap Resolution and Segmentation Accuracy}

Quantitative comparison of AdACM with a GAC (Geodesic Active Contour) [2] and the Rousson-Deriche shape based model (RD) [5] was performed (see Table 1). Overlap resolution was evaluated via: $O R=\frac{\# \text { overlaps resolved }}{\text { Total \# of overlaps }}$

\subsection{Computational Efficiency}

We evaluated the computational efficiency of AdACM with respect to a hybrid ACM (HACM) which did not employ selective invocation of the shape prior. 


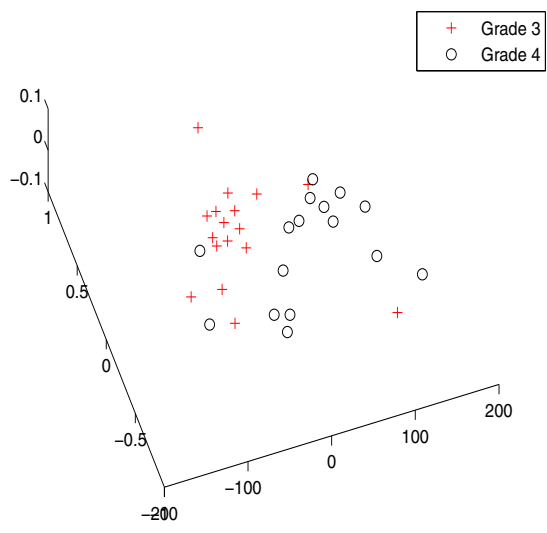

(a)

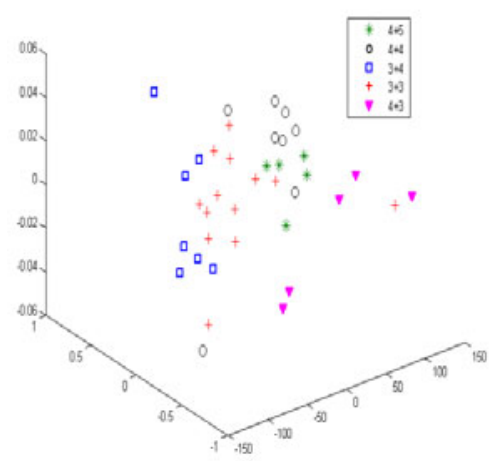

(b)

Fig. 4. PCA representation of nuclear morphologic features reveals separation of (a) primary grade 3 and grade 4 and (b) Gleason patterns $6,7,8$ and 9

Table 1. Quantitative evaluation of segmentation, and Overlap Resolution between the Geodesic Active Contour [2], Rousson-Deriche [5] and AdACM for 200 randomly selected nuclei across the 80 TMA images

\begin{tabular}{|c|c|c|c|c|}
\hline & Sensitivity & Specificity & Mean Average Distance & OR \\
\hline GAC & 0.28 & 0.92 & 7.1 & 0.04 \\
\hline RD & 0.76 & 0.88 & 1.5 & 0.74 \\
\hline AdACM & $\mathbf{0 . 8 2}$ & $\mathbf{1 . 0}$ & $\mathbf{1 . 1}$ & $\mathbf{0 . 9 0}$ \\
\hline
\end{tabular}

On image patches of $200 \times 200$ pixels and in the presence of 120 nuclei with 40 overlaps, AdACM required 250s to accurately segment all objects and resolve all intersections, compared to HACM which took 550s; all evaluations being performed on a $3 \mathrm{GHz}$, dual core processor with 4 GB RAM.

\section{Concluding Remarks}

In this work we presented a new hybrid active contour that employs a shape prior for concurrent segmentation of multiple nuclei. Our model (AdACM) selectively invokes the shape prior terms (computationally expensive step) in those image regions where objects overlap, overlaps being determined via a concavity detection scheme. This selective invocation of energy terms in the variational formulation yields a hybrid ACM that is both accurate and computationally efficient. Morphologic features derived from nuclei segmented via AdACM enabled discrimination of different Gleason grade patterns, on prostate cancer TMAs, reflecting the segmentation accuracy of AdACM. Additionally, independent segmentation 
based performance based measures also reflected the efficacy of our scheme. In future work we intend to leverage AdACM in the context of nuclear and cell segmentation in other domains within digital pathology.

\section{References}

1. Kass, M., Witkin, A., Terzopoulos, D.: Snakes: active contour models. International J. of Computer Vision, 321-331 (1987)

2. Caselles, V., Kimmel, R., Sapiro, G.: Geodesic active contours. Int.J. Comput. Vision 22(1), 61-79 (1997)

3. Chan, T., Vese, L.: Active contours without edges. IEEE Trans. on Image Processing 10(2), 266-277 (2001)

4. Fang, W., Chan, K.: Statistical Shape Influence in Geodesic Active Contours. IEEE CVPR 40(8), 2163-2172 (2007)

5. Rousson, M., Paragios, N.: Shape priors for level set representations. In: Heyden, A., Sparr, G., Nielsen, M., Johansen, P. (eds.) ECCV 2002. LNCS, vol. 2351, pp. 78-92. Springer, Heidelberg (2002)

6. Chan, T.: Level set based shape prior segmentation. IEEE CVPR 2, 1164-1170 (2005)

7. Veltri, R., Isharwal, S., Mille, M., Epstein, J.I., Partin, A.: Nuclear Roundness Variance Predicts Prostate Cancer Progression,Metastasis, and Death: A Prospective EvaluationWith up to 25 Years of Follow-Up After Radical Prostatectomy. The Prostate 70, 1333-1339 (2010)

8. Fatakdawala, H., Xu, J., Basavanhally, A., Bhanot, G., Ganesan, S., Feldman, M., Tomaszewski, J., Madabhushi, A.: Expectation Maximization driven Geodesic Active Contour with Overlap Resolution (EMaGACOR): Application to Lymphocyte Segmentation on Breast Cancer Histopathology. IEEE TBME 57(7), 1676-1689 (2010)

9. Zhang, Q., Pless, R.: Segmenting multiple familiar objects under mutual occlusion. In: $\operatorname{ICIP}(2006)$ 